Discrete Comput Geom 33:605-616 (2005)

DOI: $10.1007 / \mathrm{s} 00454-004-1094-6$

\title{
Pairs of Convex Bodies with Centrally Symmetric Intersections of Translates
}

\author{
Valeriu Soltan \\ Department of Mathematical Sciences, George Mason University, \\ 4400 University Drive, Fairfax, VA 22030-4444, USA \\ vsoltan@gmu.edu
}

\begin{abstract}
For a pair of convex bodies $K$ and $K^{\prime}$ in $E^{d}$, the $d$-dimensional intersections $K \cap\left(x+K^{\prime}\right), x \in E^{d}$, are centrally symmetric if and only if $K$ and $K^{\prime}$ are represented as direct sums $K=R \oplus P$ and $K^{\prime}=R^{\prime} \oplus P^{\prime}$ such that: (i) $R$ is a compact convex set of some dimension $m, 0 \leq m \leq d$, and $R^{\prime}=z-R$ for a suitable vector $z \in E^{d}$, (ii) $P$ and $P^{\prime}$ are isothetic parallelotopes, both of dimension $d-m$.
\end{abstract}

\section{Introduction and Main Result}

Several results of convex geometry characterize pairs of convex bodies whose intersections of translates satisfy given geometric properties. Thus, convex bodies $K$ and $K^{\prime}$ in the Euclidean space $E^{d}$ are homothetic ellipsoids if and only if for any translate $x+K^{\prime}$, $x \in E^{d}$, the intersection of the boundaries of $K$ and $x+K^{\prime}$ lies in a hyperplane (see [1]). Similarly, convex bodies $K$ and $K^{\prime}$ in $E^{d}$ are homothetic simplexes if and only if the $d$-dimensional intersections $K \cap\left(x+K^{\prime}\right), x \in E^{d}$, belong to a unique homothety class (more generally, to at most countably many homothety classes) of convex bodies (see [5]).

We study below the following problem of a similar spirit, related to centrally symmetric convex bodies.

Problem. Describe the pairs of convex bodies $K$ and $K^{\prime}$ in $E^{d}$ such that all $d$ dimensional intersections $K \cap\left(x+K^{\prime}\right), x \in E^{d}$, are centrally symmetric.

In what follows we need some definitions. A convex body is a compact convex set with nonempty interior in $E^{d}$. A set $X \subset E^{d}$ is called centrally symmetric if and only if there is a point $z \in E^{d}$ such that $X-z=z-X$; in this case $X$ is symmetric about $z$. 
We say that sets $X$ and $X^{\prime}$ in $E^{d}$ are similarly represented as direct sums

$$
X=X_{1} \oplus \cdots \oplus X_{k}, \quad X^{\prime}=X_{1}^{\prime} \oplus \cdots \oplus X_{k}^{\prime}
$$

if there are subspaces $L_{1}, \ldots, L_{k} \subset E^{d}$ forming a direct sum such that both $X_{i}$ and $X_{i}^{\prime}$ lie in $L_{i}$ for all $i=1, \ldots, k$.

A parallelotope is a compact convex set in $E^{d}$ that is a direct sum of finitely many line segments. Two parallelotopes $P$ and $P^{\prime}$ of the same dimension $k(1 \leq k \leq d)$ are called isothetic provided they can be similarly represented as direct sums

$$
P=P_{1} \oplus \cdots \oplus P_{k}, \quad P^{\prime}=P_{1}^{\prime} \oplus \cdots \oplus P_{k}^{\prime},
$$

where $P_{i}$ and $P_{i}^{\prime}$ are parallel line segments for all $i=1, \ldots, k$.

Our main result is given in the following theorem.

Theorem 1. For a pair of convex bodies $K$ and $K^{\prime}$ in $E^{d}$, the following three conditions are equivalent:

(1) All nonempty intersections $K \cap\left(x+K^{\prime}\right), x \in E^{d}$, are centrally symmetric.

(2) All d-dimensional intersections $K \cap\left(x+K^{\prime}\right), x \in E^{d}$, are centrally symmetric.

(3) $K$ and $K^{\prime}$ are similarly represented as direct sums

$$
K=R \oplus P \quad \text { and } \quad K^{\prime}=R^{\prime} \oplus P^{\prime}
$$

such that conditions (i) and (ii) below are satisfied:

(i) $R$ is a compact convex set of some dimension $m, 0 \leq m \leq d$, and $R^{\prime}=z-R$ for a suitable vector $z \in E^{d}$,

(ii) $P$ and $P^{\prime}$ are isothetic parallelotopes, both of dimension $d-m$.

Observation 1. The cases $m=d$ and $m=0$ in condition (3) of Theorem 1 are interpreted as follows: $m=d$ means that $K^{\prime}=z-K$ for a suitable vector $z \in E^{d}$, while $m=0$ means that $K$ and $K^{\prime}$ are isothetic parallelotopes, both of dimension $d$.

Corollary 1 [3]. A convex body $K \subset E^{d}$ is a parallelotope if and only if there is a real number $\lambda \in] 0,1\left[\right.$ such that all nonempty intersections $K \cap(x+\lambda K), x \in E^{d}$, are centrally symmetric.

\section{Auxiliary Theorems}

The proof of Theorem 1 is organized by induction on $d=\operatorname{dim} E^{d}$ and uses Theorems 2 and 3 below. Recall that a subset $F$ of a convex body $M \subset E^{d}$ is called an exposed face of $M$ provided there is a hyperplane $H$ supporting $M$ such that $F=M \cap H$. In what follows, $\mathcal{F}(M)$ denotes the family of exposed faces of $M$.

If an exposed face $F$ of $M$ consists of a single point (respectively, of a line segment), then it is called an exposed point (respectively, an exposed line segment). Throughout this paper we denote by $\mathcal{F}_{0}(M)$ and $\mathcal{F}_{1}(M)$ the family of exposed points and the family 
of exposed line segments of $M$, respectively. Generally, the endpoints of an exposed line segment of $M$ are not exposed points themselves, but they are extreme points of the body. Recall that a point $x \in M$ is extreme if no open line segment $] y, z[, y \neq z$, with the property $x \in] y, z[\subset M$ exists. In a standard way, the set of extreme points of $M$ is denoted ext $M$.

Let $M$ and $M^{\prime}$ be a given pair of convex bodies in $E^{d}$. For any hyperplane $H$ supporting $M$, denote by $H^{\prime}$ the hyperplane parallel to $H$ and supporting $M^{\prime}$ such that $M^{\prime}$ lies on the same side from $H^{\prime}$ as $M$ does with respect to $H$. In this case the exposed face $F^{\prime}=M^{\prime} \cap H^{\prime}$ of $M^{\prime}$ will be called associate to the exposed face $F=M \cap H$. Generally, the relation "is associate to" is not a one-to-one correspondence between the families $\mathcal{F}(M)$ and $\mathcal{F}\left(M^{\prime}\right)$.

The following theorems are auxiliary for the proof of Theorem 1. (To distinguish similarly looking elements, we use $\theta$ for the zero vector of $E^{d}$.)

Theorem 2. Let $M$ and $M^{\prime}$ be convex bodies in $E^{d}$ such that all nonempty intersections $M \cap\left(x+M^{\prime}\right), x \in E^{d}$, are centrally symmetric. Then $M$ and $-M^{\prime}$ satisfy the following two conditions:

(4) Any exposed point a of $M$ has an associate exposed point $a^{\prime}$ of $-M^{\prime}$ such that $\left(-M^{\prime}\right) \cap\left(a^{\prime}+W\right)$ is a translate of $M \cap(a+W)$ for a suitable neighborhood $W$ of $\theta$.

(5) Any exposed line segment $[a, b]$ of $M$ has an associate exposed line segment $\left[a^{\prime}, b^{\prime}\right]$ of $-M^{\prime}$ parallel to $[a, b]$ and such that for a suitable neighborhood $W$ of $\theta$ the sets $\left(-M^{\prime}\right) \cap\left(a^{\prime}+W\right)$ and $\left(-M^{\prime}\right) \cap\left(b^{\prime}+W\right)$ are translates of $M \cap(a+W)$ and $M \cap(b+W)$, respectively, provided $a-b$ and $a^{\prime}-b^{\prime}$ have the same direction.

Theorem 3. Let $M$ and $M^{\prime}$ be convex bodies in $E^{d}$. Then $M^{\prime}$ is a translate of $M$ if and only if the following two conditions are satisfied:

(6) Any exposed point a of $M$ has an associate exposed point $a^{\prime}$ of $M^{\prime}$ such that $M^{\prime} \cap\left(a^{\prime}+W\right)$ is a translate of $M \cap(a+W)$ for a suitable neighborhood $W$ of $\theta$.

(7) Any exposed line segment $[a, b]$ of $M$ has an associate exposed line segment $\left[a^{\prime}, b^{\prime}\right]$ of $M^{\prime}$ that is a translate of $[a, b]$ and such that for a suitable neighborhood $W$ of $\theta$ the sets $M^{\prime} \cap\left(a^{\prime}+W\right)$ and $M^{\prime} \cap\left(b^{\prime}+W\right)$ are translates of $M \cap(a+W)$ and $M \cap(b+W)$, respectively, provided $a-b$ and $a^{\prime}-b^{\prime}$ have the same direction.

In a standard way, bd $M$ and int $M$ denote, respectively, the boundary and the interior of a convex body $M \subset E^{d}$. A boundary point $x$ of $M$ is called regular if there is a unique hyperplane supporting $M$ at $x$. Denote by $N(M)$ the family of outward unit normals to $M$ at its regular points. In particular, $M$ is a polytope if and only if the set $N(M)$ is finite. Finally, $B_{r}(a)=\left\{x \in E^{d}:\|x-a\| \leq r\right\}$ stands for the closed ball with center $a$ and radius $r$, and $S=\left\{x \in E^{d}:\|x\|=1\right\}$ denotes the unit sphere of $E^{d}$. 


\section{Proof of Theorem 2}

We prove condition (5) only, since the proof of (4) may be considered as a limit case of (5), by taking $a=b$ and $a^{\prime}=b^{\prime}$.

Let $[a, b]$ be an exposed line segment of $M$. Translating $M$, if necessary, we may assume that $a=\theta$. Denote by $H$ a hyperplane with the property $M \cap H=[\theta, b]$, and let $e$ be the unit vector orthogonal to $H$ such that $M$ and $e$ belong to the same closed half-space $P$ determined by $H$. Let $G$ be the hyperplane through $\theta$ orthogonal to the line segment $[\theta, b]$, and let $Q$ be the closed half-space determined by $G$ and disjoint to $b$. Next, put $R=b / 2+Q$. In other words, $R$ is the closed half-space determined by the hyperplane $b / 2+G$ and containing $\theta$.

Choose a real number $\lambda>0$ so small that the hyperplane $H_{\lambda}=\lambda e+H$ intersects int $M$. Denote by $D_{\lambda}$ the part of $R$ that lies between $H$ and $H_{\lambda}$, and let $M_{\lambda}$ be the set of regular points of $M$ which lie in $D_{\lambda}$. Now let $N\left(M_{\lambda}\right)$ be the set of outward unit normals to $M$ at points from $M_{\lambda}$. Since $M \cap H=[\theta, b]$ and $R$ excludes the segment ]$b / 2, b]$, by a compactness argument we obtain the existence of a number $\delta$ such that $0<\delta<\min \{\lambda,\|b\| / 2\}$ and the closure of $N\left(M_{\delta}\right)$ belongs to an open half-sphere of the unit sphere $S$. Indeed, otherwise we would obtain the existence of two parallel hyperplanes through $\theta$ and a point from $] \theta, b$ [, respectively, both supporting $M$.

Translating $M^{\prime}$, if necessary, we may assume that $M^{\prime}$ is disjoint to int $P$ and is supported by $H$ at $\theta$ such that $M^{\prime} \cap H$ lies in $Q$. By a continuity argument, we may choose a vector $x \in D_{\delta}$ such that $\theta \in \operatorname{int}\left(x+M^{\prime}\right)$ and $P \cap\left(x+M^{\prime}\right)$ lies in $D_{\lambda}$. From the above it follows that the intersection $K=M \cap\left(x+M^{\prime}\right)$ is a convex body situated in $D_{\delta}$ and that $K \cap H=[\theta, w]$ for a point $\left.\left.w \in\right] \theta, b / 2\right]$. By the hypothesis, $K$ is centrally symmetric.

Let $F$ be the hyperplane supporting $K$ and parallel to $H, F \neq H$. Obviously, $F$ lies in the closed slab between $H$ and $x+H$. Because $K$ is centrally symmetric, there is an exposed line segment $[z, z-w]$ of $K$ with the property $K \cap F=[z, z-w]$. Moreover, $K$ is symmetric about the middle point of the line segment $[\theta, z]$.

We claim that $z \in \operatorname{int} M$. Indeed, assume, for contradiction, that $z \in \mathrm{bd} M$. Since $z \in K \subset D_{\delta}$ and $F$ intersects the interior of $M$, the boundary of $K$ in any neighborhood of $z$ should contain a $(d-1)$-dimensional piece of bd $M$. Hence any neighborhood of $z$ contains a regular point $p$ of $K$ that belongs to $M_{\delta}$. Let $e_{p}$ be the outward unit normal of $K$ (also of $M$ ) at $p$. By the symmetry of $K$ about $z / 2$, the point $q=z-p$ is a regular point of $K$ and the outward unit normal $e_{q}$ to $K$ at $q$ is opposite to $e_{p}: e_{q}=-e_{p}$. Since $\theta \in \operatorname{int}\left(x+M^{\prime}\right)$, we can choose $p$ so close to $z$ that the respective point $q$ belongs to $\operatorname{int}\left(x+M^{\prime}\right)$. As a result, $q$ lies in the boundary of $M$, and whence $q \in M_{\delta}$. Thus we have two distinct points $p, q \in M_{\delta}$ with $e_{p}=-e_{q}$, which is in contradiction with the choice of $\delta$. Hence $z \in$ int $M$.

The inclusions $z \in \operatorname{bd} K$ and $z \in \operatorname{int} M$ obviously imply that $z \in \operatorname{bd}\left(x+M^{\prime}\right)$, otherwise $z$ would lie in the interior of $K$. Moreover, the hyperplane $F$ should coincide with $x+H$. Indeed, assume for a moment that $F$ is different from $x+H$. In this case, one can find a point $u \in\left[x, z\right.$, which belongs to $M \cap\left(x+M^{\prime}\right)$ and lies between $x+H$ and $F$. The last is in contradiction with the choice of $F$.

Next we show that $z=x$. Indeed, since $z \in \operatorname{bd}\left(x+M^{\prime}\right)$ and since $x+H$ supports $K$ along the line segment $[z, z-w]$, the hyperplane $x+H$ supports $x+M^{\prime}$ along a 
line segment $[z, s]$ that contains $[z, z-w]$. Hence $M^{\prime} \cap H=[z-x, s-x]$. From the inclusion $M^{\prime} \cap H \subset Q$ and the fact that $G$ supports $M^{\prime} \cap H$ at $\theta$, we conclude that $z-x=\theta$, i. e., $z=x$. As a result, $M^{\prime} \cap H=[\theta, s-x]$, whence $[\theta, x-s]$ is an exposed line segment of $-M^{\prime}$ associate to $[\theta, b]$.

Since $\theta \in \operatorname{int}\left(x+M^{\prime}\right)$ and $x=z \in$ int $M$, there is a neighborhood $W_{1} \subset E^{d}$ of $\theta$ such that $W_{1} \subset \operatorname{int}\left(x+M^{\prime}\right)$ and $x-W_{1}$ is a neighborhood of $x$ that lies in int $M$. Because $K$ is symmetric about $x / 2$, we have

$M \cap W_{1}=K \cap W_{1}=x-K \cap\left(x-W_{1}\right)=x-\left(x+M^{\prime}\right) \cap\left(x-W_{1}\right)=\left(-M^{\prime}\right) \cap W_{1}$.

Repeating the consideration above for the points $b$ and $x-s$, we obtain the existence of a neighborhood $W_{2} \subset E^{d}$ of $\theta$ such that $(M-b) \cap W_{2}=\left(-M^{\prime}-x+s\right) \cap W_{2}$. Obviously, the set $W=W_{1} \cap W_{2}$ is a required neighborhood of $\theta$.

\section{Proof of Theorem 3}

If a convex body $M^{\prime}$ is a translate of a convex body $M$, then conditions (6) and (7) are trivially satisfied.

Conversely, let $M$ and $M^{\prime}$ be a pair of convex bodies in $E^{d}$ that satisfy conditions (6) and (7). We show that $M^{\prime}$ is a translate of $M$. This part of the proof is organized by induction on $d=\operatorname{dim} E^{d}$.

The case $d=1$ is trivial, and the case $d=2$ is based on the following statement.

Claim 1. Let $M$ and $M^{\prime}$ be convex bodies in the plane $E^{2}$ that satisfy conditions (6) and (7). Then the relation "is associate to" gives one-to-one correspondences $\mathcal{F}_{0}(M) \leftrightarrow$ $\mathcal{F}_{0}\left(M^{\prime}\right)$ and $\mathcal{F}_{1}(M) \leftrightarrow \mathcal{F}_{1}\left(M^{\prime}\right)$.

Proof of Claim 1. Choose a point $x \in \mathcal{F}_{0}(M)$, and let $x^{\prime} \in \mathcal{F}_{0}\left(M^{\prime}\right)$ be associate to $x$ such that $M^{\prime} \cap\left(x^{\prime}+W\right)$ is a translate of $M \cap(x+W)$ for a suitable neighborhood $W$ of $\theta$. Let $H$ be a line with $M \cap H=\{x\}$, and let $H^{\prime}$ be the line parallel to $H$ with $M^{\prime} \cap H^{\prime}=\left\{x^{\prime}\right\}$. Assume for a moment that $x$ has another associate point $x_{1}^{\prime} \in \mathcal{F}_{0}\left(M^{\prime}\right)$, that is, assume the existence of a line $H_{1}$ distinct from $H$ such that $M \cap H_{1}=\{x\}$ and of the line $H_{1}^{\prime}$ parallel to $H_{1}$ and supporting $M^{\prime}$ at $x_{1}^{\prime}$ only. Since $M^{\prime} \cap\left(x^{\prime}+W\right)=\left(x^{\prime}-x\right)+M \cap(x+W)$, the line $H^{\prime \prime}=x^{\prime}-x+H_{1}$ supports $M^{\prime}$ at $x^{\prime}$. Thus $H_{1}^{\prime}$ and $H^{\prime \prime}$ are parallel lines both supporting $M^{\prime}$ from the same side. As a result, $H_{1}^{\prime}=H^{\prime \prime}$ and $H_{1}^{\prime}$ supports $M^{\prime}$ along the line segment $\left[x^{\prime}, x_{1}^{\prime}\right]$, contradicting the condition $M^{\prime} \cap H_{1}^{\prime}=\left\{x_{1}^{\prime}\right\}$. Hence any exposed point $x$ of $M$ has a unique associate exposed point $x^{\prime}$ of $M^{\prime}$.

Next we prove that distinct exposed points $x_{1}$ and $x_{2}$ of $M$ have distinct associate exposed points $x_{1}^{\prime}$ and $x_{2}^{\prime}$ of $M^{\prime}$. Indeed, assume, for contradiction, that $x_{1}^{\prime}=x_{2}^{\prime}$. Let $H_{1}$, $H_{1}^{\prime}$ and $H_{2}, H_{2}^{\prime}$ be the respective pairs of parallel lines with the properties

$$
M \cap H_{1}=\left\{x_{1}\right\}, \quad M \cap H_{2}=\left\{x_{2}\right\}, \quad M^{\prime} \cap H_{1}^{\prime}=M^{\prime} \cap H_{2}^{\prime}=\left\{x_{1}^{\prime}\right\} .
$$

Let also $W_{1}$ and $W_{2}$ be some neighborhoods of $\theta$ that satisfy condition (6) for the pairs $x_{1}, x_{1}^{\prime}$ and $x_{2}, x_{1}^{\prime}$, respectively. Then the neighborhood $W=W_{1} \cap W_{2}$ of $\theta$ satisfies condition (6) for each of the pairs $x_{1}, x_{1}^{\prime}$ and $x_{2}, x_{1}^{\prime}$. As a result, both lines $H_{1}=x_{1}-$ 
$x_{1}^{\prime}+H_{1}^{\prime}$ and $H^{\prime}=x_{2}-x_{1}^{\prime}+H_{1}^{\prime}$ support $M$ such that $M \cap H_{1}=\left\{x_{1}\right\}$ and $M \cap H^{\prime}=\left\{x_{2}\right\}$. Since the lines $H_{1}$ and $H^{\prime}$ are parallel and support $M$ from the same side, they should coincide. The last is in contradiction with $x_{1} \neq x_{2}$.

Finally, let $x^{\prime} \in \mathcal{F}_{0}\left(M^{\prime}\right)$ and let $H^{\prime}$ be a line with the property $M^{\prime} \cap H^{\prime}=\left\{x^{\prime}\right\}$. Denote by $H$ the line parallel to $H^{\prime}$ and supporting $M$ such that $M$ lies on the same side from $H$ as $M^{\prime}$ does with respect to $H^{\prime}$. If $H$ supported $M$ along a line segment $[v, w]$, then $[v, w]$ would be an exposed line segment of $M$ with no associate in $\mathcal{F}_{1}\left(M^{\prime}\right)$. Hence the intersection $M \cap H$ is an exposed point $x$ of $M$. As a result, any exposed point of $M^{\prime}$ is associate to an exposed point of $M$. Summing up, we obtain that the relation "is associate to" gives a one-to-one correspondence $\mathcal{F}_{0}(M) \leftrightarrow \mathcal{F}_{0}\left(M^{\prime}\right)$.

Let $[x, z] \in \mathcal{F}_{1}(M)$, and let $\left[x^{\prime}, z^{\prime}\right] \in \mathcal{F}_{1}\left(M^{\prime}\right)$ be associate to $[x, z]$. Since the line supporting $M$ along $[x, z]$ is uniquely defined, $\left[x^{\prime}, z^{\prime}\right]$ is a unique associate to $[x, z]$. Obviously, distinct line segments from $\mathcal{F}_{1}(M)$ have distinct associate line segments from $\mathcal{F}_{1}\left(M^{\prime}\right)$.

Conversely, let $\left[x^{\prime}, z^{\prime}\right] \in \mathcal{F}_{1}\left(M^{\prime}\right)$ and let $H^{\prime}$ be the line with the property $M^{\prime} \cap H^{\prime}=$ $\left[x^{\prime}, z^{\prime}\right]$. Denote by $H$ the line parallel to $H^{\prime}$ and supporting $M$ such that $M$ lies on the same side from $H$ as $M^{\prime}$ does with respect to $H^{\prime}$. Assume for a moment that $M \cap H$ consists of a single point $v$, and let $v^{\prime} \in \mathcal{F}_{0}\left(M^{\prime}\right)$ be associate to $v$. As is easily seen, $v^{\prime}$ should coincide with one of $x^{\prime}, z^{\prime}$. Since any neighborhood of $x^{\prime}$ or $z^{\prime}$ contains a part of the line segment $\left[x^{\prime}, z^{\prime}\right]$, from (7) it follows that $M \cap H$ should contain a line segment parallel to $\left[x^{\prime}, z^{\prime}\right]$, contradicting the assumption $M \cap H=\{v\}$. Hence $\left[x^{\prime}, z^{\prime}\right]$ is associate to a line segment $[x, z] \in \mathcal{F}_{1}(M)$. Summing up, we obtain that the relation "is associate to" gives a one-to-one correspondence $\mathcal{F}_{1}(M) \leftrightarrow \mathcal{F}_{1}\left(M^{\prime}\right)$.

We continue the proof of the inductive statement for $d=2$. As is easily seen, any extreme point of a planar convex body is either an exposed point or an endpoint of an exposed line segment of the body. From Claim 1 and conditions (6) and (7) we obtain that for any extreme point $x$ of $M$ there is a unique extreme point $x^{\prime}$ of $M^{\prime}$ such that $M^{\prime} \cap\left(x^{\prime}+W_{x}\right)$ is a translate of $M \cap\left(x+W_{x}\right)$ for a suitable neighborhood $W_{x}$ of $\theta$

Denote by $\mathcal{O}(M)$ the family of open line segments ] $v, z$ such that $[v, z] \in \mathcal{F}_{1}(M)$. Obviously, the family

$$
\mathcal{C}=\mathcal{O}(M) \cup\left\{\operatorname{bd} M \cap W_{x}: x \in \operatorname{ext} M\right\}
$$

is an open cover for bd $M$. Hence bd $M$ is the union of finitely many open $\operatorname{arcs} V_{1}, \ldots$, $V_{m} \in \mathcal{C}$. From Claim 1 we conclude that the respective translates $V_{1}^{\prime}, \ldots, V_{m}^{\prime}$ of these arcs cover bd $M^{\prime}$. The last obviously implies that $M^{\prime}$ is a translate of $M$.

Assume that the inductive statement (" $M$ ' is a translate of $M$ ") is true for all $d \leq n-1$, $n \geq 3$, and let $M$ and $M^{\prime}$ be convex bodies in $E^{n}$ that satisfy conditions (6) and (7). Choose a point $a \in \mathcal{F}_{0}(M)$, and let $a^{\prime} \in \mathcal{F}_{0}\left(M^{\prime}\right)$ be associate to $a$. Translating, if necessary, we may assume that $a=a^{\prime}=\theta$, and that $L \subset E^{n}$ is an (n-1)-dimensional subspace with the property $M \cap L=M^{\prime} \cap L=\{\theta\}$ and such that both $M$ and $M^{\prime}$ lie in the same half-space of $E^{n}$ determined by $L$. Denote by $H$ and $H^{\prime}$ the hyperplanes parallel to $L$ that support $M$ and $M^{\prime}$, respectively $\left(H \neq L \neq H^{\prime}\right)$. Our goal is to show that $M=M^{\prime}$.

Let $S_{L}$ be the unit sphere of $L$, and let $G$ be the set of vectors in $S_{L}$ such that each $e \in G$ is parallel to a line segment from the set (bd $\left.M \cup \operatorname{bd} M^{\prime}\right) \backslash\left(H \cup H^{\prime}\right)$. As follows 
from [4], the (n-2)-dimensional measure of $G$ equals 0 . Hence the complementary set $F=S_{L} \backslash G$ is dense in $S_{L}$.

For any vector $e \in F$, denote by $T_{e}$ the $(n-1)$-dimensional subspace of $E^{n}$ orthogonal to $e$. Let $M_{e}$ (respectively, $M_{e}^{\prime}$ ) be the orthogonal projection of $M$ (respectively, of $M^{\prime}$ ) on $T_{e}$. Due to the choice of $F$, any boundary point of $M_{e}$ (respectively, of $M_{e}^{\prime}$ ) is the orthogonal projection of a unique boundary point of $M$ (respectively, of $M^{\prime}$ ).

Claim 2. For any $e \in F$, the orthogonal projections $M_{e}$ and $M_{e}^{\prime}$ satisfy conditions (6) and (7).

Proof of Claim 2. Let $z$ be an exposed point of $M_{e}$, and let $R$ be an $(n-2)$-dimensional affine set in $T_{e}$ with the property $M_{e} \cap R=\{z\}$. If $l(e)$ is the one-dimensional subspace of $L$ containing $e$, then $R+l(e)$ is a hyperplane in $E^{n}$ that supports $M$ at a single point, say $x$. Hence, $x$ is an exposed point of $M$. By condition (6), $M^{\prime}$ has an exposed point $x^{\prime}$ associate to $x$, and there is a neighborhood $W \subset E^{n}$ of $\theta$ such that $M^{\prime} \cap\left(x^{\prime}+W\right)$ is a translate of $M \cap(x+W)$. Denote by $z^{\prime}$ and $V$, respectively, the orthogonal projections of $x^{\prime}$ and $W$ on $T_{e}$. Then $V$ is a neighborhood of $\theta$ in $T_{e}$ such that $M_{e}^{\prime} \cap\left(z^{\prime}+V\right)$ is a translate of $M_{e} \cap(z+V)$.

Similarly, by condition (7), for any exposed line segment $[u, z]$ of $M_{e}$, the set $M_{e}^{\prime}$ contains an exposed line segment $\left[u^{\prime}, z^{\prime}\right]$ that is associate to $[u, z]$ and is a translate of $[u, z]$. If $u-z$ and $u^{\prime}-z^{\prime}$ have the same direction, then, as above, there exists a neighborhood $V$ of $\theta$ in $T_{e}$ such that $M_{e}^{\prime} \cap\left(u^{\prime}+V\right)$ is a translate of $M_{e} \cap(u+V)$ and $M_{e}^{\prime} \cap\left(z^{\prime}+V\right)$ is a translate of $M_{e} \cap(z+V)$.

By the inductive assumption, from Claim 2 it follows that $M_{e}^{\prime}$ is a translate of $M_{e}$ for any $e \in F$. Since

$$
M_{e} \cap\left(L \cap T_{e}\right)=M_{e}^{\prime} \cap\left(L \cap T_{e}\right)=\{\theta\}
$$

and both $M_{e}$ and $M_{e}^{\prime}$ lie in the same half-space of $T_{e}$ determined by its $(n-2)$-dimensional subspace $L \cap T_{e}$, we have that $M_{e}=M_{e}^{\prime}$.

Obviously, $M \subset M_{e}+l(e)$ for any $e \in F$. If $x \notin M$, then, using the density of $F$ in $S_{L}$, we can find a vector $e \in F$ such that the line $x+l(e)$ through $x$ is disjoint to $M$. Then the orthogonal projection of $x$ on $T_{e}$ does not belong to $M_{e}$, whence $x \notin M_{e}+l(e)$. Summing up, we obtain that $M=\cap\left\{M_{e}+l(e): e \in F\right\}$.

Similarly, $M^{\prime}=\bigcap\left\{M_{e}^{\prime}+l(e): e \in F\right\}$. Since $M_{e}=M_{e}^{\prime}$ for all $e \in F$, we finally have $M^{\prime}=M$.

\section{Auxiliary Lemmas}

This section contains some more auxiliary statements necessary for the proof of Theorem 1.

Lemma 1. Let $X$ be a nonempty set in $E^{d}$, and put $Y=z-X$ for some $z \in E^{d}$. Then any nonempty intersection $X \cap(x+Y), x \in E^{d}$, is symmetric about $(x+z) / 2$. 
Proof. Obviously, a set $T \subset E^{d}$ is symmetric about a point $v \in E^{d}$ if and only if $T-v$ is symmetric about $\theta$. Also, the intersection $T \cap(-T)$, if nonempty, is symmetric about $\theta$. These two observations and the equality

$$
X \cap(x+Y)-\frac{x+z}{2}=X \cap(x+z-X)-\frac{x+z}{2}=\left(X-\frac{x+z}{2}\right) \cap\left(\frac{x+z}{2}-X\right)
$$

imply that $X \cap(x+Y)$, if nonempty, is symmetric about $(x+z) / 2$.

Lemma 2. Let subspaces $L_{1}, \ldots, L_{k} \subset E^{d}$ form a direct sum, and let $S_{i}, T_{i} \subset L_{i}$ and $x_{i} \in L_{i}$ be such that $S_{i} \cap\left(x_{i}+T_{i}\right) \neq \emptyset$ for all $i=1, \ldots, k$. Put

$$
S=S_{1} \oplus \cdots \oplus S_{k}, \quad T=T_{1} \oplus \cdots \oplus T_{k}, \quad x=x_{1}+\cdots+x_{k} .
$$

Then the intersection $S \cap(x+T)$ is centrally symmetric if and only if all intersections $S_{i} \cap\left(x_{i}+T_{i}\right), i=1, \ldots, k$, are centrally symmetric.

Proof. Obviously,

$$
S \cap(x+T)=\left[S_{1} \cap\left(x_{1}+T_{1}\right)\right] \oplus \cdots \oplus\left[S_{k} \cap\left(x_{k}+T_{k}\right)\right] .
$$

If each set $S_{i} \cap\left(x_{i}+T_{i}\right)$ is symmetric about $z_{i} \in L_{i}, i=1, \ldots, k$, and $z=z_{1}+\cdots+z_{k}$, then the equality

$$
\begin{aligned}
S \cap(x+T)-z & =\left[S_{1} \cap\left(x_{1}+T_{1}\right)-z_{1}\right] \oplus \cdots \oplus\left[S_{k} \cap\left(x_{k}+T_{k}\right)-z_{k}\right] \\
& =\left[z_{1}-S_{1} \cap\left(x_{1}+T_{1}\right)\right] \oplus \cdots \oplus\left[z_{k}-S_{k} \cap\left(x_{k}+T_{k}\right)\right] \\
& =z-S \cap(x+T)
\end{aligned}
$$

implies that $S \cap(x+T)$ is symmetric about $z$.

Conversely, let the intersection $S \cap(x+T)$ be symmetric about a point $z \in E^{d}$. Clearly, $z \in L_{1} \oplus \cdots \oplus L_{k}$. Denote by $\varphi_{i}$ the parallel projection of $L_{1} \oplus \cdots \oplus L_{k}$ onto $L_{i}$ along $L_{1} \oplus \cdots \oplus L_{i-1} \oplus L_{i+1} \oplus \cdots \oplus L_{k}$, and let $z_{i}=\varphi_{i}(z), i=1, \ldots, k$. Then

$z_{i}-S_{i} \cap\left(x_{i}+T_{i}\right)=\varphi_{i}(z-S \cap(x+T))=\varphi_{i}(S \cap(x+T)-z)=S_{i} \cap\left(x_{i}+T_{i}\right)-z_{i}$.

Hence each set $S_{i} \cap\left(x_{i}+T_{i}\right)$ is symmetric about $z_{i}, i=1, \ldots, k$.

Lemma 3. Let $X_{1}, X_{2}, \ldots$ be a sequence of centrally symmetric compact sets in $E^{d}$ convergent in the Hausdorff metric to a bounded set $X$. Then the limit set $X$ is also centrally symmetric.

Proof. Let $X_{i}$ be symmetric about a point $z_{i}, i=1,2, \ldots$ Since $X_{i} \rightarrow X$, all the sets $X_{i}$ are situated in a neighborhood of $X$, and, as a result, the sequence $z_{1}, z_{2}, \ldots$ is bounded. If $z_{i_{1}}, z_{i_{2}}, \ldots$ is a subsequence of $z_{1}, z_{2}, \ldots$ that converges to a point $z$, then

$$
X-z=\lim _{j \rightarrow \infty}\left(X_{i_{j}}-z_{i_{j}}\right)=\lim _{j \rightarrow \infty}\left(z_{i_{j}}-X_{i_{j}}\right)=z-X,
$$

i.e., $X$ is symmetric about $z$. 


\section{Proof of Theorem 1}

First we prove the equivalence of conditions (1) and (2).

Since (1) obviously implies (2), it is sufficient to show that (2) $\Rightarrow$ (1). Let $X=$ $K \cap\left(x+K^{\prime}\right), x \in E^{d}$, be nonempty, and choose a point $y \in X$. Then there is a sequence $y_{1}, y_{2}, \ldots$ of points from int $K$ that converges to $y$. Consider the intersections $X_{i}=K \cap\left(x+y_{i}-y+K^{\prime}\right), i=1,2, \ldots$ Since $y_{i} \in($ int $K) \cap\left(x+y_{i}-y+K\right)$, each set $X_{i}$ has dimension $d$. By (2), all $X_{i}$ are centrally symmetric. Since $X_{i} \rightarrow X$ in the Hausdorff metric when $i \rightarrow \infty, X$ is centrally symmetric itself (see Lemma 3 ).

The remaining part of the proof is devoted to the equivalence of conditions (1) and (3). Lemmas 1 and 2 above obviously imply that (3) $\Rightarrow$ (1). Hence, it remains to show that $(1) \Rightarrow(3)$. Since the case $d=1$ is trivial, we assume that $d \geq 2$. If $K^{\prime}=z-K$ for a suitable vector $z \in E^{d}$, we have finished the proof. Assume that $K^{\prime} \neq z-K$ for any $z \in E^{d}$. Then Theorems 2 and 3 imply the existence of an exposed line segment $[a, b]$ of $K$ that has an associate exposed line segment $\left[a^{\prime}, b^{\prime}\right]$ of $-K^{\prime}$ such that $\left[a^{\prime}, b^{\prime}\right]$ is not a translate of $[a, b]$. By a symmetry argument, we may assume that $\left\|a^{\prime}-b^{\prime}\right\|<\|a-b\|$.

Translating $K$ and $-K^{\prime}$, if necessary, we may assume that $a=a^{\prime}=\theta$. Condition (1) implies the existence of a hyperplane $H$ supporting both $K$ and $-K^{\prime}$ such that $K$ and $-K^{\prime}$ lie in the same closed half-space $P$ determined by $H$, with $K \cap H=[\theta, b]$ and $\left(-K^{\prime}\right) \cap H=\left[\theta, b^{\prime}\right]$, where $\left.b^{\prime} \in\right] \theta, b[$. Moreover, there is a neighborhood $W$ of the origin $\theta$ such that $K \cap W=\left(-K^{\prime}\right) \cap W$. Hence for any point $x \in$ int $K \cap W$, the intersection $K \cap\left(x+K^{\prime}\right)$ is a convex body, centrally symmetric about $x / 2$ (see Lemma 2). In particular, $H$ supports $K \cap\left(x+K^{\prime}\right)$ along a line segment $\left.\left.[\theta, c], c \in\right] \theta, b\right]$, and the hyperplane $x+H$ supports $K \cap\left(x+K^{\prime}\right)$ along the line segment $[x, x-c]$. Moreover, $\theta \in \operatorname{int}\left(x+K^{\prime}\right)$, as shown in the proof of Theorem 2 .

Denote by $l$ the line containing the segment $[\theta, b]$, and let $l_{x}$ be the line through $x$ parallel to $l$. By a continuity argument, the point $x$ above can be chosen so close to $\theta$ that the line segment $l_{x} \cap K$ becomes arbitrarily close to $[\theta, b]$; in particular, $l_{x} \cap K$ becomes longer than $\left[\theta, b^{\prime}\right]$. Thus we can translate $x+K^{\prime}$ along the line $l_{x}$ into a position $x+w+K^{\prime}, w \in l$, such that the exposed line segment $\left[x+w, x+w-b^{\prime}\right]$ of the body $x+w+K^{\prime}$ lies in int $K$.

Claim 3. For any points $z \in] \theta, b\left[\right.$ and $\left.z^{\prime} \in\right] \theta, b^{\prime}[$, there is a neighborhood $W$ of $\theta$ such that $\left(-K^{\prime}\right) \cap\left(z^{\prime}+W\right)$ is a translate of $K \cap(z+W)$.

Proof of Claim 3. First we choose $z^{\prime}$ to be the middle point of $\left[x+w, x+w-b^{\prime}\right]$. Since $K \cap\left(x+w+K^{\prime}\right)$ is centrally symmetric, we obtain that the point $z \in[\theta, b]$ symmetric to $z^{\prime}$ satisfies the conclusion of Claim 3. Shifting the body $x+w+K^{\prime}$ both ways along the line $l_{x}$ such that $\left[x+w, x+w-b^{\prime}\right]$ remains in $K$, and using the symmetry of intersections $K \cap\left(x+w+K^{\prime}\right)$, we obtain that any point $u \in[\theta, b]$ from a small neighborhood of $z$ satisfies, together with $z^{\prime}$, the conclusion of Claim 3. Coming back to $z^{\prime}$, we obtain that any point $u \in\left[x+w, x+w-b^{\prime}\right]$ from a small neighborhood of $z^{\prime}$, satisfies, together with $z$, the conclusion of Claim 3. Continuing along this way, we get the proof of Claim 3.

Claim 3 implies the following corollary. 
Corollary 2. For any points $z \in] \theta, b\left[\right.$ and $\left.z^{\prime} \in\right] \theta, b^{\prime}[$, the generated cones

$C_{z}(K)=\{z+\lambda(x-z): x \in K, \lambda \geq 0\}, \quad C_{z^{\prime}}\left(K^{\prime}\right)=\left\{z^{\prime}+\lambda\left(x-z^{\prime}\right): x \in K^{\prime}, \lambda \geq 0\right\}$

satisfy the relation $C_{z^{\prime}}\left(K^{\prime}\right)+z^{\prime}=z-C_{z}(K)$, and each of these cones contains the line $l$.

Claim 4. The line segment $\left[\theta,-b^{\prime}\right]$ is an affine diameter of $K^{\prime}$, i.e., there are distinct parallel hyperplanes through $\theta$ and $-b^{\prime}$, respectively, both supporting $K^{\prime}$.

Proof of Claim 4. Equivalently, $\left[x+w, x+w-b^{\prime}\right]$ is stated to be an affine diameter of $x+w+K^{\prime}$. It is known (see, e.g., [2]) that a chord [r,s] of a convex body $C \subset E^{d}$ is an affine diameter of $C$ if and only if $[r, s]$ is a longest chord of $C$ in the direction parallel to $[r, s]$.

Assume, for contradiction, that $\left[x+w, x+w-b^{\prime}\right]$ is not an affine diameter of $x+w+K^{\prime}$. Then there exists a line segment $[p, q] \subset\left(x+w+K^{\prime}\right)$ parallel to $l$ and longer than $\left[x+w, x+w-b^{\prime}\right]$. By a continuity argument, we may consider that ]$p, q\left[\subset \operatorname{int}\left(x+w+K^{\prime}\right)\right.$. Then the relative interior of the trapezoid $A$ with bases $\left[x+w, x+w-b^{\prime}\right]$ and $[p, q]$ lies in the interior of $x+w+K^{\prime}$. Due to Corollary 2 , we may choose the point $x \in$ int $K \cap W$ and the respective point $w \in l$ such that $A$ intersects $l$ along a line segment $\left[p_{1}, q_{1}\right]$ that lies inside $[\theta, b]$. Obviously, the hyperplanes $x+H$ and $H$ support the symmetric convex body $K \cap\left(x+w+K^{\prime}\right)$ along the line segments $\left[x+w, x+w-b^{\prime}\right]$ and $\left[p_{1}, q_{1}\right]$, respectively, a contradiction with the fact that $\left[p_{1}, q_{1}\right]$ is longer than $\left[x+w, x+w-b^{\prime}\right]$. Thus $\left[x+w, x+w-b^{\prime}\right]$ is a longest chord of $x+w+K^{\prime}$ in the direction $l$, whence it is an affine diameter of $x+w+K^{\prime}$.

Claim 5. There is a hyperplane $T$ through $\theta$ and not containing $l$ such that $K^{\prime}$ has a pair of $(d-1)$-dimensional exposed faces parallel to $T$ and containing the points $\theta$ and $-b^{\prime}$, respectively.

Proof of Claim 5. Since $\left[\theta,-b^{\prime}\right]$ is an affine diameter of $K^{\prime}$, there is a hyperplane $T$ supporting $K^{\prime}$ that passes through $\theta$ and does not contain $l$ such that the hyperplane $T-b^{\prime}$ also supports $K^{\prime}$. We prove that the sets $K^{\prime} \cap T$ and $K^{\prime} \cap\left(T-b^{\prime}\right)$ are the required $(d-1)$-dimensional exposed faces of $K^{\prime}$.

First we show the existence of a neighborhood $V$ of the point $t=-b^{\prime} / 2$ such that the line segment $(z+l) \cap K^{\prime}$ is of length at least $\left\|b^{\prime}\right\|$ for any point $z \in K^{\prime} \cap V$. An obvious modification of the considerations preceding Claim 3 implies the existence of a point $x \in K$ close to $-t$ and of a point $w \in l$ such that $\left[w+x, w+x-b^{\prime}\right]$ lies in $K$. Moreover, Claim 3 implies the existence of a neighborhood $U$ of $-t$ such that $\left[w+x, w+x-b^{\prime}\right] \subset K$ for all $x \in K \cap U$. Furthermore, $U$ can be chosen such that $\left(-K^{\prime}\right) \cap U=K \cap U$. Then each intersection $K \cap\left(x+w+K^{\prime}\right), x \in K \cap U$, is centrally symmetric and is supported by the hyperplane $x+H$ along the line segment $\left[x+w, x+w-b^{\prime}\right]$. Hence each $K \cap\left(x+w+K^{\prime}\right), x \in K \cap U$, is supported by $H$ along a line segment $\left[r, r-b^{\prime}\right]$ that lies in $l$ and is a translate of $\left[x+w, x+w-b^{\prime}\right]$. Obviously, the line segment $\left[r-x-w, r-x-w-b^{\prime}\right]$ is of length $\left\|b^{\prime}\right\|$ and lies in 
$K^{\prime} \cap(l-x-w)=K^{\prime} \cap(l-x)$. Therefore, the segment $K^{\prime} \cap(l-x)$ is of length at least $\left\|b^{\prime}\right\|$. Finally, put $V=-U$.

On the other hand, $\left[\theta,-b^{\prime}\right]$ is a longest chord of $K^{\prime}$ in the direction $l$. Hence the line segment $(z+l) \cap K^{\prime}$ is exactly of length $\left\|b^{\prime}\right\|$ for any point $z \in K^{\prime} \cap V$. Since each such segment lies between the parallel hyperplanes $T$ and $T-b^{\prime}$, its endpoints lie on $T$ and $T-b^{\prime}$, respectively. Obviously, these endpoints fill some $(d-1)$-dimensional sets in $T$ and $T-b^{\prime}$, respectively. Thus both sets $K^{\prime} \cap T$ and $K^{\prime} \cap\left(T-b^{\prime}\right)$ are $(d-1)$ dimensional.

Claim 6. $K$ and $K^{\prime}$ are similarly represented as direct sums $K=Q \oplus[\theta, b]$ and $K^{\prime}=Q^{\prime} \oplus\left[\theta, b^{\prime}\right]$, where $Q$ and $Q^{\prime}$ are $(d-1)$-dimensional compact convex sets in $T$.

Proof of Claim 6. In view of Corollary 2 and Claim 5, it is sufficient to prove that for any two-dimensional plane $L$ through $l$, both intersections $P=K \cap L$ and $P^{\prime}=K^{\prime} \cap L$, if two-dimensional, are isothetic parallelograms.

According to the consideration above, $P$ is supported by the line $l$ along the segment $[\theta, b]$, and $P^{\prime}$ is supported by $l$ along the line segment $\left[\theta,-b^{\prime}\right]$. Moreover, $P^{\prime}$ is supported by the lines $R=L \cap T$ and $R-b^{\prime}$ along some segments $\left[\theta, v^{\prime}\right]$ and $\left[-b^{\prime}, w^{\prime}\right]$, respectively, where $T$ is the hyperplane defined in Claim 5.

Since any nonempty intersection $K \cap\left(x+K^{\prime}\right)$ is supported by the hyperplanes $H$ and $x+H$ along the line segments $[\theta, w] \subset l$ and $[x, x-w]$, respectively, and since both these segments lie in $L$, we conclude that for any point $x \in L$ the set $P \cap\left(x+P^{\prime}\right)$ equals $L \cap K \cap\left(x+K^{\prime}\right)$. Hence $P \cap\left(x+P^{\prime}\right), x \in L$, is centrally symmetric if and only if $K \cap\left(x+K^{\prime}\right)$ is centrally symmetric.

To show that $P$ and $P^{\prime}$ are isothetic parallelograms, we consider only those intersections $P \cap\left(x+P^{\prime}\right), x \in L$, which are parallelograms, and, as a consequence, derive the respective properties of the boundaries of $P$ and $P^{\prime}$. For simplicity, our considerations are performed in the plane $L$, such that both $P$ and $P^{\prime}$ have nonempty interior.

Choose a point $x \in$ int $P$ such that $\left[x, x-v^{\prime}\right]$ intersects $[\theta, b]$ and $\left[x, x-b^{\prime}\right]$ intersects the boundary of $P$. From the central symmetry of $P \cap\left(x+P^{\prime}\right)$ we conclude that $P \cap\left(x+P^{\prime}\right)$ has to be a parallelogram. Then bd $P$ contains a line segment $[\theta, v]$ that lies in $R$. By a similar argument, bd $P$ contains a line segment $[b, w] \subset b+R$.

Considering the possible cases $\|v\|<\left\|v^{\prime}\right\|,\|v\|>\left\|v^{\prime}\right\|,\|v\|=\left\|v^{\prime}\right\|$, we first assume that $\|v\|<\left\|v^{\prime}\right\|$. Then there is a scalar $\lambda>0$ such that $\lambda v+x+P^{\prime}$ entirely contains $[\theta, v]$ and $\left[\lambda v+x, \lambda v+x-v^{\prime}\right]$ still intersects $[\theta, b]$. Since $P \cap\left(\lambda v+x+P^{\prime}\right)$ is a parallelogram, bd $P$ contains a line segment $[v, u]$ parallel to $l$. Moving $\lambda v+x+P^{\prime}$ further along the ray $\{\lambda v: \lambda>0\}$ and looking for the intersection of $P$ and $\lambda v+x+P^{\prime}$, we obtain that bd $P^{\prime}$ contains a line segment $\left[v^{\prime}, u^{\prime}\right]$ parallel to $l$. Now moving $v+P^{\prime}$ along the ray $\{\lambda b: \lambda>0\}$ and, if necessary, again along the ray $\{\lambda v: \lambda>0\}$, we obtain that $\|u-v\|$ is at least $\left\|b^{\prime}\right\|$, and $P^{\prime}$ is a parallelogram. Further movement of $v+P^{\prime}$ along the ray $\{\lambda v: \lambda>0\}$ gives us that $P$ is also a parallelogram isothetic to $P^{\prime}$.

In a similar way, any of the cases $\|v\|>\left\|v^{\prime}\right\|,\|w\|>\left\|w^{\prime}\right\|,\|w\|<\left\|w^{\prime}\right\|$ gives us that $P$ and $P^{\prime}$ are isothetic parallelograms. It remains to assume that $\|v\|=\left\|v^{\prime}\right\|$ and $\|w\|=\left\|w^{\prime}\right\|$. Then moving $b^{\prime}+P^{\prime}$ along the ray $\{\lambda v: \lambda>0\}$ we get that $w=v$ and $w^{\prime}=v^{\prime}$, i.e., that $P$ and $P^{\prime}$ are isothetic parallelograms. 
We finalize the proof of Theorem 1 by induction on $d$. The case $d=2$ is confirmed in the proof of Claim 6. Assume that (1) $\Rightarrow$ (3) is true for all $d \leq n-1, n \geq 2$, and let convex bodies $K, K^{\prime} \subset E^{n}$ satisfy condition (1). By Claim 6, $K$ and $K^{\prime}$ are similarly represented as direct sums $K=Q \oplus[\theta, b]$ and $K^{\prime}=Q^{\prime} \oplus\left[\theta, b^{\prime}\right]$, where $Q$ and $Q^{\prime}$ are $(n-1)$-dimensional compact convex sets in the $(n-1)$-dimensional subspace $T$ of $E^{n}$. Obviously, $Q \cap\left(x+Q^{\prime}\right), x \in T$, is nonempty if and only if $K \cap\left(x+K^{\prime}\right)$ is nonempty. By condition (1), every nonempty intersection $Q \cap\left(x+Q^{\prime}\right), x \in T$, is centrally symmetric, and, by the inductive assumption, $Q$ and $Q^{\prime}$ satisfy condition (3), with $n-1$ instead of $d$. From $K=Q \oplus[\theta, b]$ and $K^{\prime}=Q^{\prime} \oplus\left[\theta, b^{\prime}\right]$ we obviously conclude that $K$ and $K^{\prime}$ satisfy condition (3), with $n$ instead of $d$.

\section{Acknowledgment}

The author thanks the referee for many helpful comments on an earlier draft of this paper.

\section{References}

1. P. R. Goodey, Homothetic ellipsoids, Math. Proc. Cambridge Philos. Soc. 93 (1983), 25-34.

2. P. C. Hammer, Convex curves of constant Minkowski breadth, in Convexity (V. Klee, ed.), pp. 291-304, American. Mathematical. Society., Providence, RI, 1963.

3. A. B. Kharazishvili, Characterization properties of a parallelepiped, Soobshch. Akad. Nauk. Gruzin. SSR 72 (1973), 17-19.

4. D. G. Larman and C. A. Rogers, Increasing paths on the one-skeleton of a convex body and the directions of line segments on the boundary of a convex body, Proc. London Math. Soc. 23 (1971), 683-694.

5. V. Soltan, A characterization of homothetic simplices, Discrete Comput. Geom. 22 (1999), 193-200.

Received October 22, 2003, and in revised form January 21, 2004. Online publication June 21, 2004. 